Int. J. Dev. Biol. 59: 479-485 (2015)

doi: $10.1387 / \mathrm{ijdb} .150086 \mathrm{pr}$

\title{
Conditional pulmonary over-expression of Claudin 6 during embryogenesis delays lung morphogenesis
}

\author{
FELIX R. JIMENEZ, SAMUEL T. BELGIOUE, JOSHUA B. LEWIS, SCOTT A. ALBRIGHT, CAMERON M. JONES, \\ BRIAN M. HOWELL, ALEKSANDER P. MIKA, TYSON R. JERGENSEN, JASON R. GASSMAN, RYAN J. MORRIS, \\ JUAN A. ARROYO and PAUL R. REYNOLDS*
}

\author{
Lung and Placenta Research Laboratory, Brigham Young University, Physiology and Developmental Biology, \\ Provo, Utah, USA
}

\begin{abstract}
Claudin 6 (Cldn6) is a tetraspanin protein expressed by barrier epithelial cells. In order to assess the effects of persistent tight junctions involving Cldn6 during lung development, a doxycycline (dox)-inducible conditional transgenic mouse was generated that up-regulates Cldn6 in the distal lung. Pups had unlimited access to dox from conception until sacrifice date at embryonic day (E) 18.5. Quantitative PCR, immunoblotting, and immunohistochemistry revealed significantly elevated Cldn6 expression in transgenic mice compared to non-transgenic controls. There were no differences in terms of lung size, lung weight, or whole body weight at the time of necropsy. Histological evaluations led to the discovery that E18.5 Cldn6 transgenic pups appeared to be in the early canalicular stage of development coincident with fewer, thickened respiratory airspaces. In contrast, controls appeared to have entered the saccular stage characterized by thin airspace walls and spherical architecture. Immunostaining for transcriptional regulators including TTF-1 and FoxA2 was conducted to assess cell differentiation and specific cell types were identified via staining for pro-surfactant protein C (alveolar type II epithelial cells) or Clara Cell Secretory Protein (cub or Clara cells). Lastly, cell turnover was qualitatively measured via staining for cell proliferation or apoptosis. These data suggest that Cldn6 is an important junctional protein potentially involved in the programming of epithelial cells during lung development. Furthermore, genetic down-regulation of Cldn6 as development proceeds may influence differentiation observed in the transition from the canalicular to the saccular lung.
\end{abstract}

KEY WORDS: claudin 6, lung, transgenic, mouse

Lung development is a complex process of highly organized and dynamic events. Tubular branching of the lung airways characterizes the pseudoglandular stage of lung organogenesis and the subsequent canalicular stage coincides with pulmonary epithelial cell differentiation that results in the formation of the air-blood barrier (Burri, 1984; Torday, 1992; Copland and Post, 2004). Numerous signaling and transcriptional control pathways critically influence the precise deposition of specialized cell types along the proximaldistal pulmonary axis.

Tight junctions (TJs) are increasingly recognized as potentially critical modulators spatial epithelial cell programming. TJs begin to form at cell-cell contacts as the respiratory epithelium develops into a complex monolayer (Crapo et al., 1983; Ward and Nicholas, 1984). TJs are critical in the developing lung as they provide the means of compartmentalization required of barrier derivation. TJS are an assembly of resident integral proteins in the membranes of neighboring cells, a collection of diverse accessory proteins, and the cytoskeleton (Aijaz et al., 2006; Balda and Matter, 2000). These junctional structures rely on transmembrane proteins such as occludin, junctional adhesion molecules (JAMs), and a family of tetraspanin molecules called Claudins (Cldns) primarily responsible for establishing robust cell-cell contact (Aijaz et al., 2006; Chiba et al., 2008; Krause et al., 2008). This highly conserved family of proteins is composed of 27 members (Mineta et al., 2011) that produce a variety of tight junctions and thus influence barrier epithelium and characteristic permeability (Anderson, 2006; Morita

Abbreviations used in this paper: CCSP, clara cell secretory protein; Cldn6, claudin 6; Dox, doxycycline; FoxA2, Forkhead Box A2; PCNA, proliferating cell nuclear antigen; SP-C, surfactant protein C; TJ, tight junction; TTF-1, thyroid transcription factor-1.

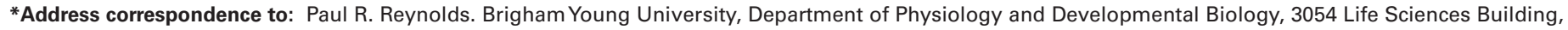
Provo, UT 84602, USA.Tel: +1-(801)422-1933. Fax: +1-(801)422-0700. E-mail: paul_reynolds@byu.edu
}

Accepted: 4 June 2015.

ISSN: Online 1696-3547, Print 0214-6282 
A

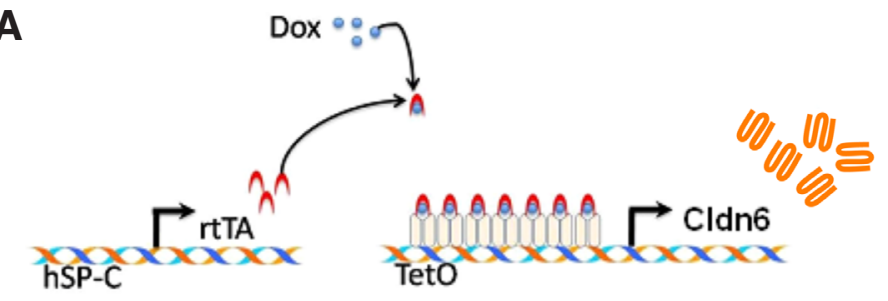

B
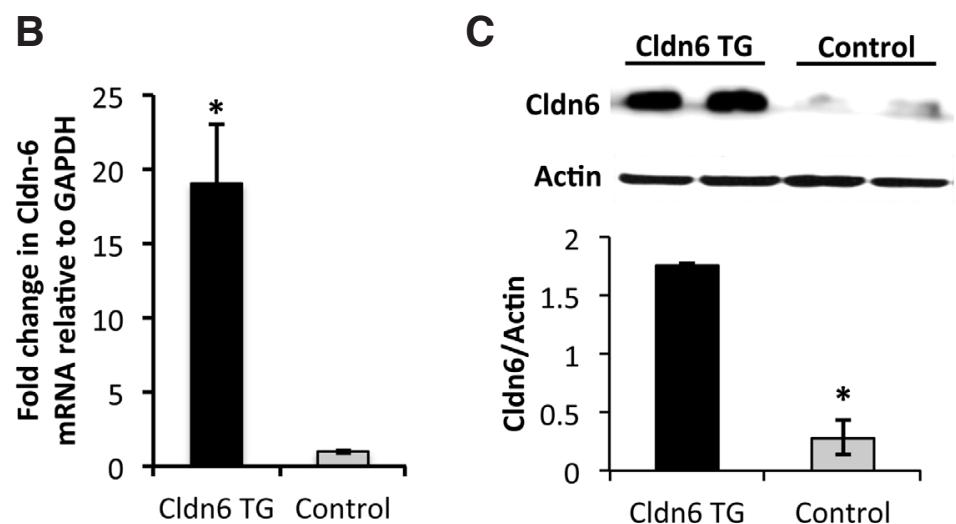
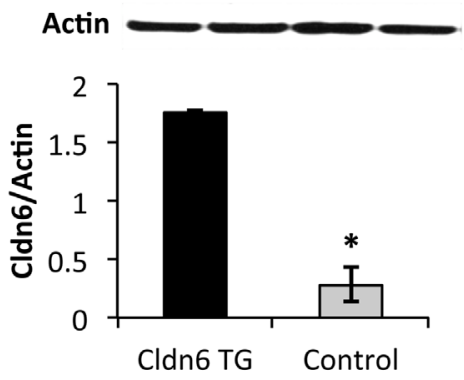

et al., 1999). Of this family, Claudin-6 (Cldn6) is identified to be crucial for epithelial cell differentiation and permeability during early embryonic development (Troy and Turksen, 2007; Turksen and Troy, 2002). In addition to occlusion, a key function for Cldn6 is to also regulate sodium and chloride permeability via the specific geometry of extracellular loops (EL) in the protein's secondary and tertiary structures (Van Itallie and Anderson, 2006; Yu et al., 2009). During murine lung morphogenesis, Cldn6 is highly expressed between embryonic day $(E) 10.5$ to $E$ 16.5. A general theme exists wherein higher expression occurs during early stages of lung development with diminished expression as lung morphogenesis proceeds (Jimenez et al., 2014; Troy et al., 2009; Turksen and Troy, 2002).

Given the diversity and complexity of Cldn family members, conditional transgenic overexpression and knock-down models

Fig. 1. Cldn6 TG mice up-regulated Cldn6. (A) Doxycycline (dox)inducible expression of Cldn6 in double transgenic mice. The rtTA protein was expressed using the human SP-C (hSP-C) promoter active in respiratory epithelium. In the presence of dox, rtTA induced the expression of Cldn6 in lung epithelium. (B) qPCR for Cldn6 mRNA revealed significantly increased expression in Cldn6 TG mice compaed to controls. Fold changes are presented relative to GAPDH expression. (C) Immunoblotting demonstrated significantly increased Cldn6 protein expression in Cldn6 TG mice. Densitometry is presented as ratios of Cldn6 protein intensity divided by actin used as a loading control. Immunoblotting and qPCR data are representative of experiments performed in triplicate and statistical differences are noted $\left({ }^{*} P \leq 0.05\right)$.

seem to be suitable for testing hypotheses related to tissuespecific roles of individual Cldn members in vivo. Indeed, such approaches have been demonstrated when determining the contributions of Cldn6 in deriving epidermal models and when dissecting trophectoderm formation (Moriwaki et al., 2007). In this research, we evaluated lung morphogenesis in transgenic mice that utilized the human promoter for surfactant protein-C (hSP-C) to conditionally overexpress Cldn6 in the respiratory compartment (Perl and Whitsett, 1999). The data demonstrate remarkable delays in lung morphogenesis coincident with impaired cell turnover. The data further suggest a role for Cldn6 in the orchestration of perinatal cell differentiation characteristic of maturing pulmonary airspaces. Understanding the role of Cldn6 should shed light on the functions of TJs during lung development and in conditions where compromised lung development predisposes individuals to hypoplastic lung complications.

\section{Results}

\section{Cldn6 expression was up-regulated in the lungs of Cldn6 TG mice}

Cldn6 is steadily down-regulated as later stages of lung morphogenesis are encountered (Felix paper 1). Therefore, an inducible, lung specific Cldn6 TG mouse was generated in order to test the hypothesis that persistent Cldn6 impairs normal lung epithelial cell biology (Reynolds et al., 2010). A human surfactant protein C (hSP-C) promoter was used to express a reverse tetracycline transactivator (rtTA) in alveolar epithelial cells. Double transgenic mice contained this transgene as well as a second transgene that included TetO response elements upstream of the Cldn6 gene (Cldn6 TG, Fig. 1A). Dox administration from conception until sacrifice date at E18.5 revealed a significant increase in the transcription of the Cldn6 gene, as revealed by quantitative RT-PCR of RNA isolates from Cldn6 TG mouse lungs and age-matched controls (Fig. 1B). Protein translation of significantly increased

Fig. 2. Cldn6 TG mice expressed increased Cldn6. Cldn6 was detected by immunohistochemistry in epithelial cells of Cldn6 TG mice, but only minimally detected in the lungs of non-transgenic control litter mates. Qualitative staining for Cldn6 revealed staining in the mid sized airways (arrow) and only minimal expression in the larger airways (arrowhead). Images are representative of at least 3 different animals per group and the original magnification of images was at 20X or 40X as indicated. 


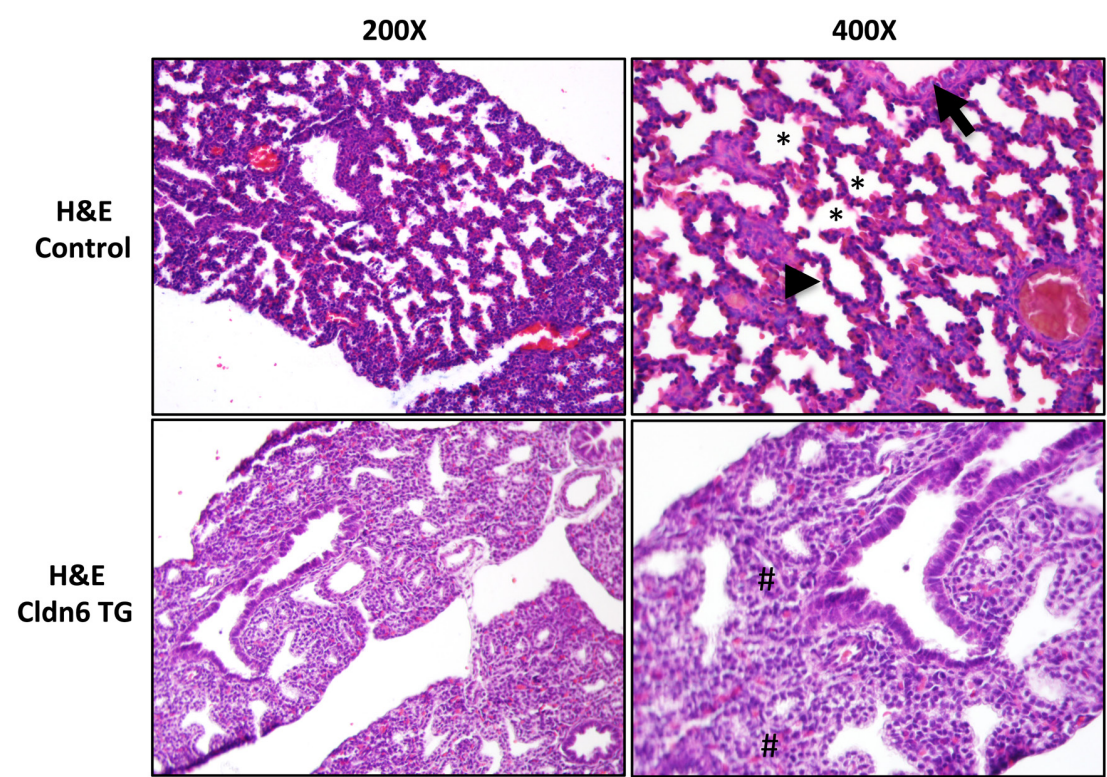

Fig. 3. Cldn6 TG mice expressed perturbed lung morphology. Hematoxylin-eosin staining of lungs from Cldn6 TG mice showed persistence of the cannalicular stage. In particular, prolific distal airspaces in control mouse lungs (*) were lacking in the Cldn6 TG mouse. Controls expressed clear ciliated columnar cells (arrow) and thinning epithelium in the maturing distal lung (arrowhead). A key characteristic of the Cldn6 TG muse lung was persistent thickened mesenchyme (\#). Images are representative of at least 3 different animals per group and the original magnification of images was at 20X or 40X as indicated.

Cldn6 mRNA expression was confirmed by immunoblotting. Lung lysates from Cldn6 TG mice expressed significantly more Cldn6 protein when compared to controls (Fig. 1C).

Immunohistochemical analysis was next performed in order to spatially assess elevated Cldn6 in the lungs of Cldn6 TG mice. Cldn6 was only minimally detected in the lungs of E18.5 control mice (Fig. 2). Immunostaining performed with Cldn6 TG mouse lung sections revealed robust, intense staining for Cldn6 in the developing epithelial lining of primitive airways throughout the distal lung (Fig. 2). Specifically, Cldn6 expression tended to dominate mid sized airways (Fig. 2, arrow) as opposed to the most proximal airways (Fig. 2, arrowhead). These data demonstrated that Cldn6 is qualitatively and quantitatively increased in the lungs of Cldn6 TG mice.

\section{Cldn6 up-regulation delayed lung development in Cldn6 TG mice}

In comparison to controls, morphological alterations in the proximal-distal patterning of the Cldn6 TG mouse lung were discovered following classic hematoxylin and eosin (H\&E) staining (Fig. 3). Specifically, the numerous distal airspaces observed in 20X and 40X lung images from control lungs (Fig. $\left.3,{ }^{\star}\right)$ were lacking in images obtained from Cldn6 TG mouse lungs. Control mouse lungs displayed stark differences in ciliated columnar epithelium (Fig. 3, arrow) and cuboidal/squamous epithelium in the distal lung (Fig. 3, ar- rowhead). Conversely, lungs from Cldn6 TG mice contained a mostly tall cuboidal/columnar epithelial cell phenotype and significantly thickened mesenchymal deposition (Fig. 3, \#). In summary, control lungs contained thinner alveolar septa, flattened epithelium, and expanding saccules; however, Cldn6 TG mice exhibited fewer and larger lung spaces with distinctly dense intersaccular mesenchyme. These morphological data suggested delays in the maturation of Cldn6 TG mouse lungs and a hypoplastic canalicular phenotype at a period normally characteristic of the late saccular lung.

To further investigate pulmonary maturation, lungspecific transcriptional regulators were evaluated by immunohistochemistry in the lungs of Cldn6 TG and control mice. Thyroid transcription factor (TTF)-1, also known as Nkx2.1, is a homeodomain-containing transcription factor that is critical for normal lung development. TTF-1 expression was intensely observed in both Cldn6 TG and control mice (Fig. 4). However, TTF-1 tended to be diffusely expressed in both the proximal and distal lung compartments in control lungs, but more intensely expressed by airway epithelium in the lungs of Cldn6 TG mice (Fig. 4). TTF-1 is known to partner with Forkhead box A2 (FoxA2) in the coordination of target gene expression during cell differentiation (Maeda et al., 2007). FoxA2, which is expressed during lung cell commitment and maturation, was expressed sporadically by epithelium in both the proximal (Fig. 5, arrow) and distal lung (Fig. 5, arrowhead). Interestingly, FoxA2, a key TTF-1 co-regulator, was almost absent in the Cldn6 TG mouse lung (Fig. 5). These findings related to perturbed expression of critical transcription factors support the notion that Cldn6 TG mice are developmentally delayed.

\section{Cldn6 up-regulation impaired proximal and distal lung cell differentiation}

To further phenotypically characterize the potential delays in the Cldn6 TG mouse lung, two pulmonary epithelial cell-specific markers were assessed by immunohistochemistry. Clara Cell

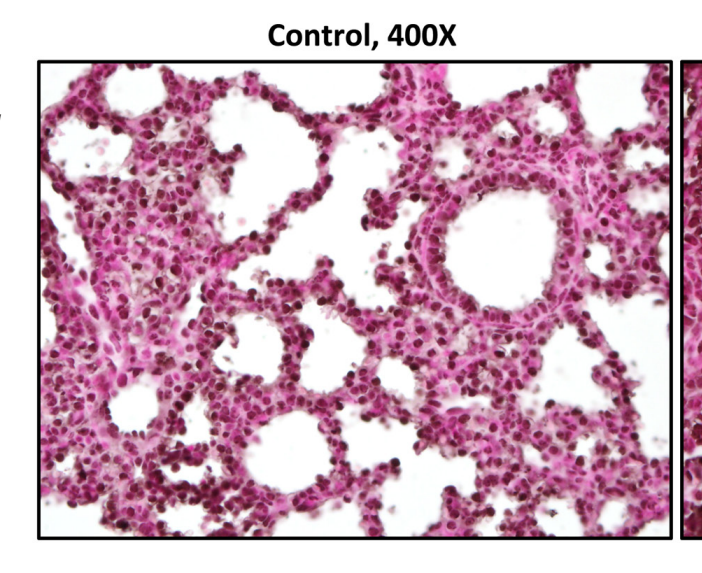

Fig. 4. TTF-1 was expressed by epithelium in both the Cldn6TG and control mouse lung. TTF-1 positive cells were found diffusely throughout the respiratory compartment of control mouse lungs; however, TTF-1 was primarily expressed by airway epithelial cells in the Cldn6TG mouse. Images are representative of at least 3 different animals per group and the original magnification of images was at 40X as indicated. 
Secretory Protein (CCSP) and surfactant protein C (SP-C) are targets of TTF-1/FoxA2 transcriptional control programs and they are key proteins expressed by proximal club cells and distal lung epithelial cells, respectively. CCSP is specifically expressed in non-ciliated epithelial cells of the airway while SP-C is expressed by differentiated alveolar type II cells. As anticipated, marked CCSP expression was detected in the larger airways of controls mice (Fig. 6, arrow); however, CCSP was significantly diminished in the airways of Cldn6 TG mice (Fig. 6 ). SP-C was assessed by staining for its proprotein (proSPC). SP-C was detected in numerous differentiating pulmonary epithelial cells in the distal mouse lung of control mice (Fig. 6, arrowhead), but restricted to the persistently larger airways in the Cldn6 TG mouse lung (Fig. 6). Abnormal expression of key epithelial cell markers provide additional evidence for delayed pulmonary morphogenesis in Cldn6 TG mice.

\section{Cldn6 up-regulation caused a cell proliferation/apoptosis imbalance in Cldn6 TG mice}

Because the Cldn6 TG mouse lung appeared morphologically delayed (Fig. 3) and cell differentiation was potentially impaired Figs. 4-6), we next assessed cell turnover by screening markers for proliferation and apoptosis. Immunochemistry was used to assess Proliferating Cell Nuclear Antigen (PCNA), which is a marker of DNA synthesis detectible during the S-phase of a mitotically active cell. As expected, proliferation coincident with PCNA expression was observed in the control mouse lung (Fig. 7), but only minimally detected in pulmonary epithelial cell populations in the Cldn6 TG mouse (Fig. 7). Interestingly, PCNA activity was not detected in the robust mesenchyme that persisted in the E18.5 Cldn6 TG mouse lung (Fig. 7). Active caspase-3 was also evaluated by immunofluorescence in order to evaluate casp-3-mediated cell death. Apoptosis controlled by active casp-3 was observed in the lungs of control mice (Fig. 8), but diminished in the lungs of Cldn6 TG mice. No active casp-3 was observed in control mouse lungs incubated in serum lacking primary antibody (Fig. 7, Neg control).

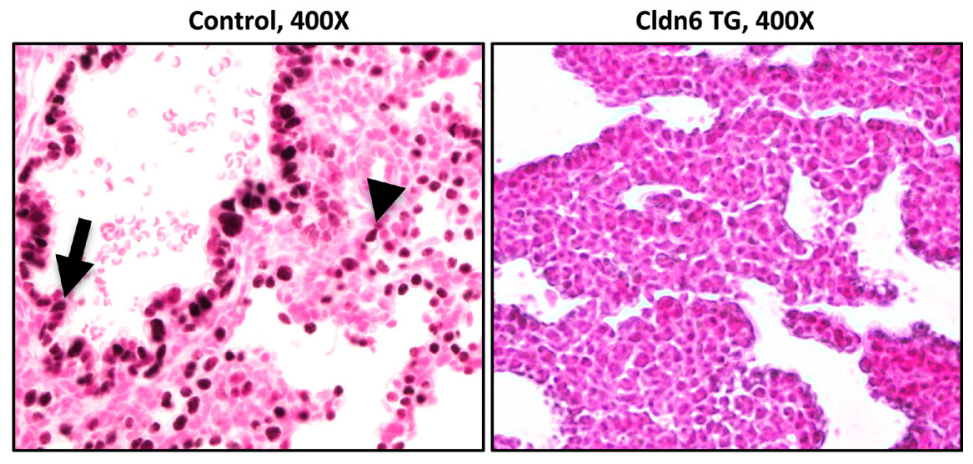

Fig. 5. FoxA2 was not detected in the lungs of CIdn6 TG mice. FoxA2 partners with TTF-1 in order to orchestrate specific gene expression programs necessary for differentiating pulmonrya epithelial cell types. FoxA2 was observed in larger airways of control mice (arrow) and distal lung epithelial cells (arrowhead) in the control mice. However, FoxA2 was nearly absent in the Cldn6 TG mouse lung. Images are representative of at least 3 different animals per group and the original magnification of images was at $40 \mathrm{X}$ as indicated.

Taken together, these data demonstrate decreased cell proliferation and apoptosis in Cldn6 TG mice as likely causes for delayed morphogenesis observed in CLdn6 TG when compared to controls.

\section{Discussion}

Diverse signaling and transcriptional control mechanisms influence regulative processes that pattern epithelial cell deposition along the proximal-distal pulmonary axis. Such pathways include those associated with fibroblast growth factors (Fgfs), sonic hedgehog (Shh), bone morphogenetic protein 4 (Bmp4), vascular endothelial growth factors (Vegfs), thyroid transcription factor 1 (TTF-1), and Wnts (Burri, 1984; Cardoso, 2001; Copland and Post, 2004; deMello et al., 1997; Gluck, 1978; Stogsdill et al., 2012; Ten Have-Opbroek, 1991; Torday, 1992). When these or other important genetic pathways are defective or delayed, pulmonary hypoplasia
CCSP IHC, 400X

Control
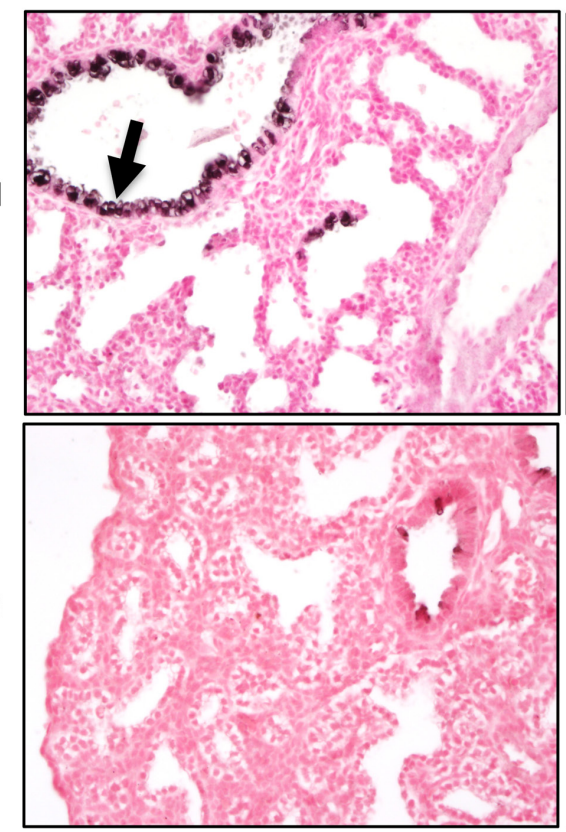

ProSP-C, 400X

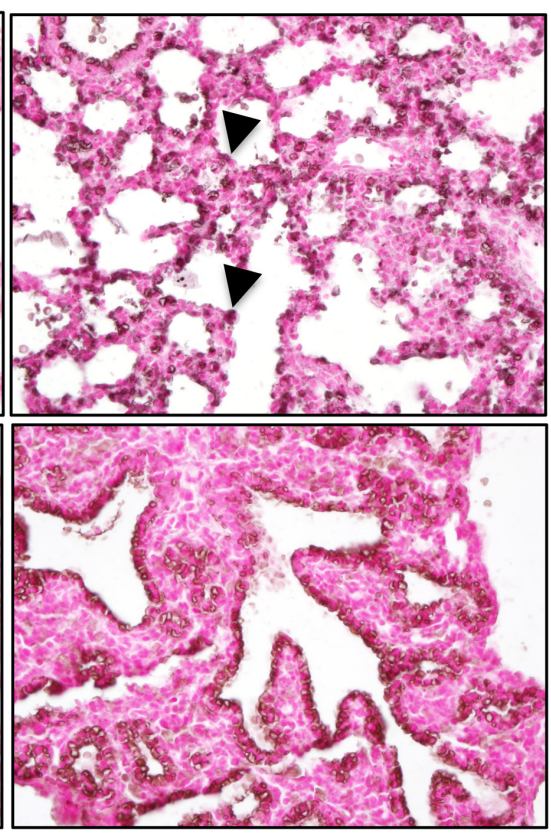

Fig. 6. Cldn6TG mice had altered expression of proximal and distal lung cell markers. Clara Cell Secretory Protein (CCSP) was detected in the proximal airways of control mice (arrow), but only a paucity of staining was observed in the airways of Cldn6 TG mice. Staining for the proprotein of surfactant protein $C$ (proSP-C) revealed diffuse staining in the parenchyma of control mice (arrowhead), but proSP-C localization in Cldn6 TG mice was primarily observed in the lining of respiratory airways. Images are representative of at least 3 different animals per group and the original magnification of images was at $40 X$ as indicated. 

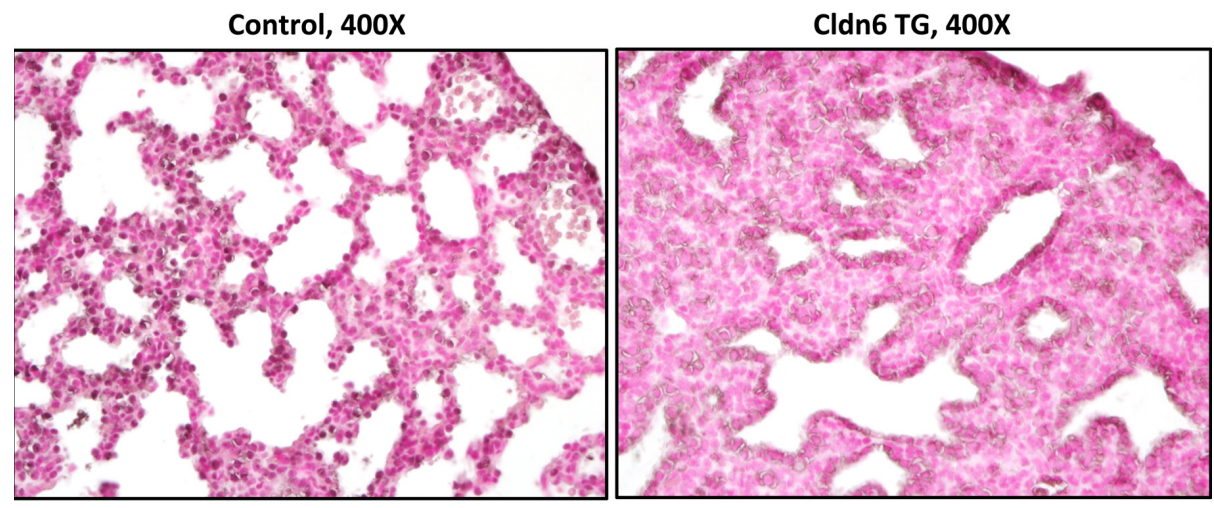

Fig. 7. Lung cell proliferation was diminished in Cldn6TG mice. Staining for Proliferating Cell Nuclear Antigen (PCNA) revealed highly distributed proliferating cells in control lungs and a near absence of proliferation in the lungs of Cldn6 TG mice. Images are representative of at least 3 different animals per group and the original magnification of images was at $40 \mathrm{X}$ as indicated. or pulmonary agenesis may occur resulting in abnormally low or absent bronchopulmonary segments and terminal alveoli (Ballard, 1980; Stogsdill et al., 2013).

A key discovery in the current investigation is that persistent, up-regulated Cldn6 positioned between pulmonary epithelial cells causes a developmentally delayed phenotype. These data offer intriguing insight into the potential roles Cldn6 has in the orchestration of branching morphogenesis and cell commitment observed during lung formation. In fact, Cldn6 expression may not only provide barrier integrity during lung organogenesis, but it may also influence the trajectory of terminal cell type differentiation and thus permanently impair normal lung formation.

Defects in lung development that present perinatally are often

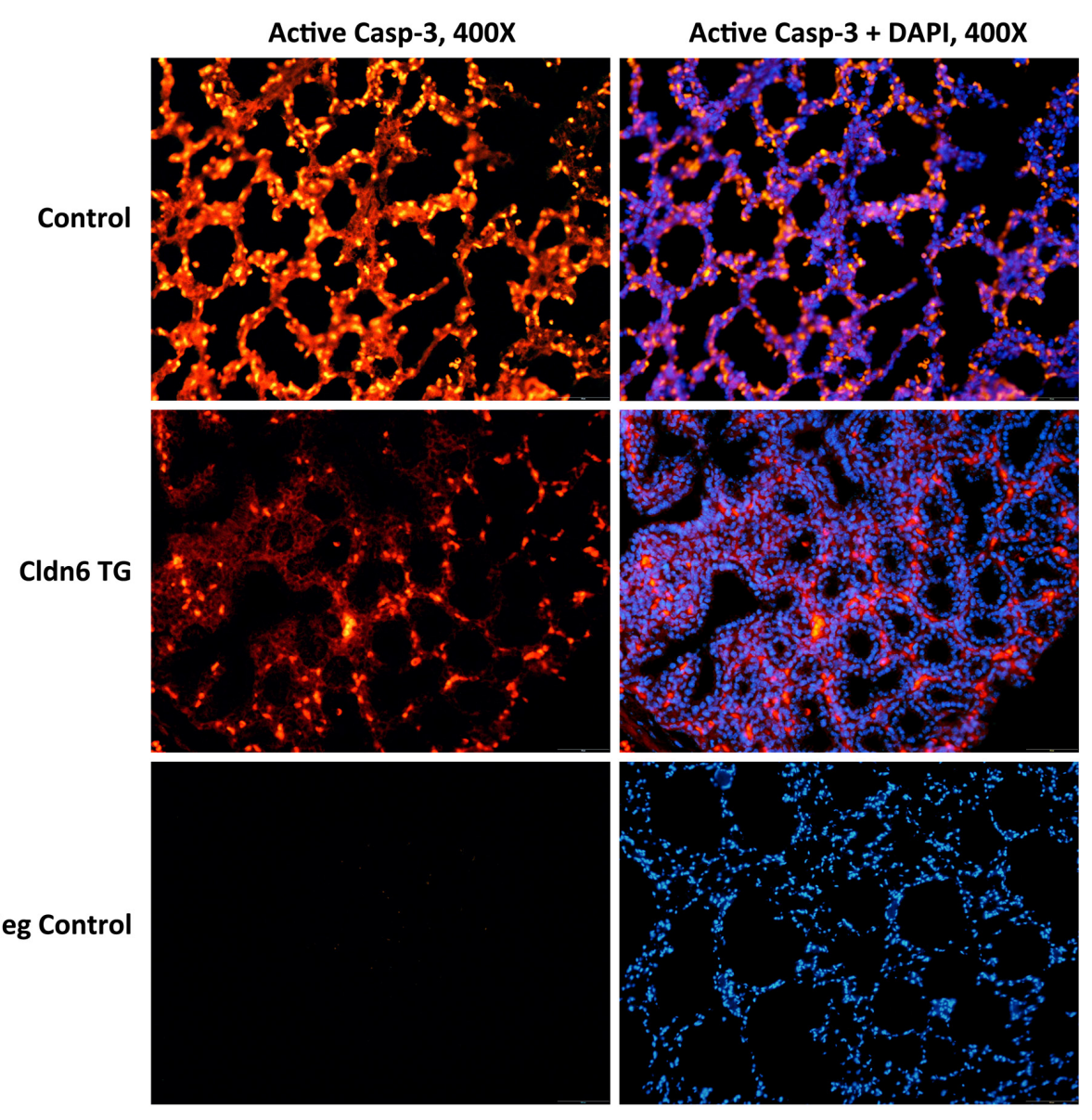

caused by delayed maturation of the alveolar compartment. A common neonatal condition associated with alveolar hindrance is bronchopulmonary dyplasia (BDP). Specifically, BPD has been characterized by alveolar growth disorders (Chambers and van Velzen, 1989; Rojas et al., 1995) and growth impairment leads to long-term diffuse reduction in the number of alveoli and gasexchange surface area (Husain et al., 1998; Jobe, 1999). Adult lung injury often results in a growth-arrested lung; however, BPD occurs in growing lungs that have unresolved morphogenetic events. Our research involving highly expressed Cldn6 during lung formation implies a role for TJs in primitive barrier cell populations that transition from one lung developmental period to the next. The formation of definitive alveoli through the process of secondary septation and mesenchymal thinning is fundamentally a postnatal event; however, uncompleted development prior to alveologenesis may be a key factor in compromising later developmental periods (Burri, 1997). Neonates susceptible to BPD are generally born in the early saccular phase, or even in the canalicular phase of lung development for those that are most premature (Burri, 1997). Several points during development have been implicated in terms of the pathophysiological mechanisms that lead to BPD including hindered cell proliferation (Jankov and Tanswell, 2004), inflammation and fibrotic process (Speer, 2003), or oxidative stress (Saugstad, 2003). Of particular relevance is the work reviewed by Jankov et al., that details diminished cellular availability as a causal aspect of lung prematurity and BPD. Our research suggests Cldn6 may influence hindered cell abundance via reductions in both proliferation and apoptosis that ensure

Fig. 8. Lung cell apoptosis was diminished in Cldn6 TG mice. Active caspase 3 (Casp-3) expres sion in control mouse lungs was detected by immunofluorescence and observed to be highly expressed in the control mouse lung. In comparison to controls, active casp-3 was decreased in the lungs of Cldn6 TG mice. No expression was observed in control lung sections incubated without primary anti-casp-3 IgG (Neg control). Images are representative of at least 3 different animals per group and the original magnification of images was at 40X as indicated. 
cell stagnation in the transgenic lung.

A review of the literature identifies possible in vivo models that reproduce lung prematurity similar to BPD. The most relevant model related to the human condition is the baboon that has been prematurely delivered and ventilated (Coalson et al., 1999). Rodents experience alveologenesis from post-natal days 4-20 and neonatal exposure to hyperoxia inhibits septal formation in a fashion similarly observed in BPD (Warner et al., 1998). The present investigation does not purport to establish a novel, working model of BPD, rather our research uncovers previously unknown contributions of Cldn6 in the temporal progression of lung development and maturation.

Diminished staining for TTF-1 and FoxA2, factors implicit in pulmonary epithelial cell refinement, suggested that elevated Cldn6 influences the transcriptional regulation of lung cell differentiation. We have already published in vitro research that specifies Cldn6 regulatory programs mediated by TTF-1, Gata-6, and FoxA2 (Jimenez et al., 2014). This work expands the impact of previous in vitro work by demonstrating potential feedback between these factors and Cldn6 availability in vivo. TTF-1 regulates target gene expression in concert with other gene regulatory factors to fine tune lung branching morphogenesis and to coordinate the expression of homeostatic proteins such as surfactant proteins (Boggaram, 2009; Bohinski et al., 1994; Hösgör et al., 2002). In addition, FoxA2 is required for normal airway epithelial differentiation and lung organogenesis (Wan et al., 2004). Thus, Cldn6 augmentation during lung organogenesis may directly or indirectly impact fundamental processes including cell population expansion and differentiation (Jimenez et al., 2014).

In summary, susceptibility to impaired branching morphogenesis and lung maturation are features of premature lung disease and other causes of respiratory distress. Our ability to imitate aspects of premature lungs through the genetic up-regulation of Cldn6, a tetraspanin protein involved in TJ assembly, significantly elevates the roles for TJs in the developing lung. Although the results presented in the current publication detail ramifications of Cldn6 over-expression during highly plastic periods of lung embryogenesis, Cldn6 up-regulation should also be carefully screened in the adult mouse. Additional studies in the adult lung that center on increased Cldn6 expression and mechanisms of inflammatory disease, epithelial permeability, and repair processes that rely on proliferation may provide promising targets for intervention.

\section{Materials and Methods}

\section{Mice}

Two transgenic lines in a C57Bl/6 background were generated and mated to create conditional doxycycline (dox)-inducible mice that overexpress Cldn6 (Fig. 1A). Select Dams were fed dox (625 mg/kg; Harlan Teklad, Madison, WI) from before conception until E18.5. En block lungs were harvested and fixed in $4 \%$ paraformaldehyde for histological analysis. Tail biopsies were genotyped by PCR for existence of transgenes using the following primers: SP-C-rtTAforward (5'-GACACATATAAGACC CTG GTC A-3') and reverse (5'-AAAATC TTG CCAGCTTTC CCC-3') and TetO-Cldn6 forward (5'-GAA TTC ATG GCC TCT ACT GGT CTG CA-3') and reverse (5'-TCT AGA TCA CAC ATA ATT CTT GGT GGG A-3'). PCR conditions included $95^{\circ} \mathrm{C}$ for 2 minutes and 35 cycles at $95^{\circ} \mathrm{C}$ for $30 \mathrm{~s}, 62^{\circ} \mathrm{C}$ (SP-C) or $56^{\circ} \mathrm{C}$ (Cldn6) for $30 \mathrm{~s}$, and $72^{\circ} \mathrm{C}$ for $45 \mathrm{~s}$. Mice were housed and utilized in accordance with protocols approved by the IACUC at Brigham Young University and at least six mice were included in each group.

\section{Histology and immunohistochemistry}

Cldn6 TG and non-transgenic control lungs (E18.5) were fixed in 4\% paraformaldehyde, processed, embedded and sectioned at $5 \mu \mathrm{m}$ thickness (Reynolds et al., 2004). Hematoxylin and eosin (H\&E) staining was performed to observe general lung morphology. To perform immunostaining for specific markers and immunoflorescence, slide samples were dehydrated, deparaffinized, processed with antigen retrieval by citrate buffer, and incubated with primary and secondary antisera that utilize HRP conjugation with Vectors Elite Kit (Vector Laboratories; Burlingame, CA) (Reynolds et al., 2003; Reynolds et al., 2011; Stogsdill et al., 2012). Antibodies that were used include: Anti-Cldn6 goat polyclonal antibody (C-20, 1:100; Santa Cruz Biotechnologies, Santa Cruz, CA), proliferating cell nuclear antigen (PCNA, SC-7907, 1:500; Santa Cruz Biotechnology), thyroid transcription factor 1 (TTF-1, WRAB-1231, 1:100; Seven Hills BioReagents, Cincinnati, OH), forkhead box A2 (FoxA2, WRAB-1200, 1:100; Seven Hills BioReagents), Clara cell secretory protein (CCSP, WRAB-3950, 1:100; Seven Hills BioReagents), and Surfactant Protein- C (SP-C, WRAB-76694, 1:100; Seven Hills BioReagents).

\section{Immunofluorescence}

Immunofluorescent detection of cleaved Caspase 3 (Casp3, PA5-16335, 1:100; Cell Signaling, Beverly, MA) was performed on $5 \mu \mathrm{m}$ thick lung sections. Slides were incubated overnight with a rabbit cleaved Caspase 3 primary antibody. Sections were then incubated with anti-rabbit fluorescein conjugated secondary antibodies for 1 hour. 4',6-diamidino-2-phenylindole, dihydrochloride (DAPI) was used for nuclear counterstaining. Slides were viewed on a fluorescence microscope with the appropriate excitation and emission filter.

\section{Immunoblotting}

Tissues were homogenized in protein lysis buffer (RIPA, Fisher Scientific, Pittsburg, PA). Protein lysates $(20 \mu \mathrm{g})$ were separated on Mini-PROTEAN $\AA$ TGX ${ }^{\text {TM }}$ Precast gel (Bio-Rad Laboratories, Inc) by electrophoresis and transferred to nitrolcellulose membranes. Membranes were blocked and incubated with a goat polyclonal antibody against Cldn6 (at a dilution of 1:200; Santa Cruz Biotechnology). A secondary donkey anti-goat immunoglobin (lg)-horseradish peroxidase antibody (1:10,000, Santa Cruz Biotechnology) was incubated in $5 \%$ milk for one hour at room temperature. The membranes were incubated with chemiluminescent substrate (Pierce, Rockford, IL) for 5 minutes and the emission of light was digitally recorded by using a C-DiGit@ Blot Scanner (LI-COR, Inc, Lincoln, Nebraska). Quantification of Cldn 6 was performed by densitometry and normalization with actin provided comparisons between Cldn6 TG and control lung samples.

\section{qRT-PCR}

Total RNA was isolated from mouse lungs using an RT-PCR Miniprep Kit (Stratagene, La Jolla, CA). Reverse transcription of RNA was performed using the Invitrogen Superscript III First-Strand Synthesis System (Life Technologies, Grand Island, NY) in order to obtain cDNA for qRT-PCR. The following primers were synthesized by Invitrogen Life Technologies (Grand Island, NY): Cldn6 (For-GCA GTC TCT TTT GCA GGC TC and Rev-CCC AAG ATT TGC AGA CCA GT) and GAPDH (For-TAT GTC GTG GAG TCT ACT GGT and Rev-GAG TTG TCA TAT TTC TCG TGG). The cDNA amplification and data analysis were performed using Bio Rad iQ SYBR Green Supermix (Bio-Rad Laboratories, Hercules, CA) and a Bio Rad Single Color Real Time PCR detection system (Bio-Rad Laboratories) (Robinson et al., 2012).

\section{Statistical analysis}

Data were assessed by one- or two-way analysis of variance (ANOVA). When ANOVAindicated significant differences, the Student'st-test was used with the Bonferroni correction for multiple comparisons. The results presented are representative, and $P$ values $\leq 0.05$ were considered significant. 


\section{Acknowledgments}

Dr. Jeffrey A. Whitsett at the Cincinnati Children's Hospital Medical Center kindly provided the hSPC-rtTA mice critical for these studies. The authors also acknowledge a team of undergraduates at Brigham Young University including Paul J. Baker for assistance in histology experiments and Jared S. Bodine, Dallin Millner, and Derek Marlor for invaluable assistance with mouse husbandry and molecular mouse identification.

\section{References}

AIJAZ, S., BALDA, M.S. and MATTER, K. (2006). Tight junctions: molecular architecture and function. Int Rev Cytol 248: 261-298.

ANDERSON, C.M.V.I.A.J.M. (2006). Claudins and epithelial paracellular transport. Annu Rev Physiol 68: 403-429.

BALDA, M.S. and MATTER, K. (2000). Transmembrane proteins of tight junctions. Semin Cell Dev Biol 11: 281-289.

BALLARD, P.L. (1980). Hormonal influences during fetal lung development. Ciba Found Symp 78: 251-274.

BOGGARAM, V. (2009). Thyroid transcription factor-1 (TTF-1/Nkx2. 1/TITF1) gene regulation in the lung. Clinical Science 116: 27-35.

BOHINSKI, R.J., DI LAURO, R. and WHITSETT, J.A. (1994). The lung-specific surfactant protein $B$ gene promoter is a target for thyroid transcription factor 1 and hepatocyte nuclear factor 3 , indicating common factors for organ-specific gene expression along the foregut axis. Molec. Cell. Biol. 14: 5671-5681.

BURRI, P. (1997). Structural aspects of prenatal and postnatal development and growth of the lung. Lung growth and development. New York: Marcel Dekker1-35.

BURRI, P.H. (1984). Fetal and postnatal development of the lung. Annu Rev Physiol 46: 617-628.

CARDOSO, W.V. (2001). Molecular regulation of lung development. Annu Rev Physiol 63: 471-494.

CHAMBERS, H.M. and VAN VELZEN, D. (1989). Ventilator-related pathology in the extremely immature lung. Pathology 21: 79-83.

CHIBA, H., OSANAI, M., MURATA, M., KOJIMA, T. and SAWADA, N. (2008). Transmembrane proteins of tight junctions. Biochim Biophys Acta 1778: 588-600.

COALSON, J.J., WINTER, V.T., SILER-KHODR, T. and YODER, B.A. (1999). Neonatal chronic lung disease in extremely immature baboons. Am. J. Resp. Crit. Care Med. 160: 1333-1346.

COPLAND, I. and POST, M. (2004). Lung development and fetal lung growth. Paediatr Respir Rev 5 Suppl A: S259-S264.

CRAPO, J., YOUNG, S., FRAM, E., PINKERTON, K., BARRY, B. and CRAPO, R. (1983). Morphometric characteristics of cells in the alveolar region of mammalian lungs. Am Rev Respir Dis 128: S42-S46.

DEMELLO, D.E., SAWYER, D., GALVIN, N. and REID, L.M. (1997). Early fetal development of lung vasculature. Am J Respir Cell Mol Biol 16: 568-581.

GLUCK, L. (1978). Fetal lung development. Mead Johnson Symp Perinat Dev Med40-49.

HÖSGÖR, M., IJZENDOORN, Y., MOOI, W.J., TIBBOEL, D. and DE KRIJGER, R.R. (2002). Thyroid transcription factor-1 expression during normal human lung development and in patients with congenital diaphragmatic hernia. J. Pediatric Surg. 37: 1258-1262.

HUSAIN, A.N., SIDDIQUI, N.H. and STOCKER, J.T. (1998). Pathology of arrested acinar development in postsurfactant bronchopulmonary dysplasia. Hum. Pathol. 29: 710-717.

JANKOV, R.P. and TANSWELL, A.K. (2004). Growth factors, postnatal lung growth and bronchopulmonary dysplasia. Paediatric Resp. Rev. 5: S265-S275.

JIMENEZ, F.R., LEWIS, J.B., BELGIQUE, S.T., WOOD, T.T. and REYNOLDS, P.R. (2014). Developmental lung expression and transcriptional regulation of Claudin-6 by TTF-1, Gata-6, and FoxA2. Resp. Res. 15: 70.

JOBE, A.J. (1999). The new BPD: an arrest of lung development. Pediatric Res. 46: 641-641.

KRAUSE, G., WINKLER, L., MUELLER, S.L., HASELOFF, R.F., PIONTEK, J. and BLASIG, I.E. (2008). Structure and function of claudins. Biochim Biophys Acta 1778: 631-645.

MAEDA, Y., DAVÉ, V. and WHITSETT, J.A. (2007). Transcriptional control of lung morphogenesis. Physiological Rev. 87: 219-244.

MINETA, K., YAMAMOTO, Y., YAMAZAKI, Y., TANAKA, H., TADA, Y., SAITO, K., TAMURA, A., IGARASHI, M., ENDO, T., TAKEUCHI, K. et al., (2011). Predicted expansion of the claudin multigene family. FEBS Lett 585: 606-612.

MORITA, K., FURUSE, M., FUJIMOTO, K. and TSUKITA, S. (1999). Claudin multigene family encoding four-transmembrane domain protein components of tight junction strands. Proc Natl Acad Sci USA 96: 511-516.

MORIWAKI, K., TSUKITA, S. and FURUSE, M. (2007). Tight junctions containing claudin 4 and 6 are essential for blastocyst formation in preimplantation mouse embryos. Dev. Biol. 312: 509-522.

PERL, A. and WHITSETT, J. (1999). Molecular mechanisms controlling lung morphogenesis. Clin Genet 56: 14-27.

REYNOLDS, P., MUCENSKI, M. and WHITSETT, J. (2003). Thyroid Transcription Factor (TTF) -1 Regulates the Expression of Midkine (MK) during Lung Morphogenesis. Dev Dyn 227: 227-237.

REYNOLDS, P., STOGSDILL, J., STOGSDILL, M. and HEIMANN, N. (2011). Upregulation of RAGE by alveolar epithelium influences cytodifferentiation and causes severe lung hypoplasia. Am J Respir Cell Mol Biol 45: 1195-1202.

REYNOLDS, P.R., MUCENSKI, M.L., LECRAS, T.D., NICHOLS, W.C. and WHITSETT, J.A. (2004). Midkine is regulated by hypoxia and causes pulmonary vascular remodeling. J. Biol. Chem. 279: 37124-37132.

ROBINSON, A.B., JOHNSON, K.D., BENNION, B.G. and REYNOLDS, P.R. (2012). RAGE signaling by alveolar macrophages influences tobacco smoke-induced inflammation. Am. J. Physiol-Lung Cell. Molec. Physiol. 302: L1192-L1199.

ROJAS, M.A., GONZALEZ, A., BANCALARI, E., CLAURE, N., POOLE, C. and SILVA-NETO, G. (1995). Changing trends in the epidemiology and pathogenesis of neonatal chronic lung disease. J. Pediatrics 126: 605-610.

SAUGSTAD, O.D. (2003). Bronchopulmonary dysplasia-oxidative stress and antioxidants. In Sem. Neonatology, vol. 8. Elsevier, pp. 39-49.

SPEER, C.P. (2003). Inflammation and bronchopulmonary dysplasia. In Sem. Neonatology, vol. 8. Elsevier, pp. 29-38.

STOGSDILL, J., STOGSDILL, M., PORTER, J., HANCOCK, J., ROBINSON, A. and REYNOLDS, P. (2012). Embryonic over-expression of RAGE by alveolar epithelium induces an imbalance between proliferation and apoptosis. Am J Respir Cell Mol Biol 47: 60-66.

STOGSDILL, M.P., STOGSDILL, J.A., BODINE, B.G., FREDRICKSON, A.C., SEFCIK, T.L., WOOD, T., KASTELER, S. and REYNOLDS, P.R. (2013). Conditional RAGE over expression in the adult murine lung causes airspace enlargement and induces inflammation. Am J Resp Cell Mol Biol 49: 128-134.

TEN HAVE-OPBROEK, A.A. (1991). Lung development in the mouse embryo. Exp Lung Res 17: 111-130.

TORDAY, J. (1992). Cellular timing of fetal lung development. Semin Perinatol 16: 130-139.

TROY, T.C., ARABZADEH, A., LARIVIERE, N.M.K., ENIKANOLAIYE, A. and TURKSEN, K. (2009). Dermatitis and Aging-Related Barrier Dysfunction in Transgenic Mice Overexpressing an Epidermal-Targeted Claudin 6 Tail Deletion Mutant. Plos One 4.

TROY, T.C. and TURKSEN, K. (2007). The targeted overexpression of a Claudin mutant in the epidermis of transgenic mice elicits striking epidermal and hair follicle abnormalities. Mol Biotechnol 36: 166-174.

TURKSEN, K. and TROY, T.C. (2002). Permeability barrier dysfunction in transgenic mice overexpressing claudin 6. Development 129: 1775-1784.

VAN ITALLIE, C.M. and ANDERSON, J.M. (2006). Claudins and epithelial paracellular transport. Annu. Rev. Physiol. 68: 403-429.

WAN, H., KAESTNER, K.H., ANG, S.-L., IKEGAMI, M., FINKELMAN, F.D., STAHLMAN, M.T., FULKERSON, P.C., ROTHENBERG, M.E. and WHITSETT, J.A. (2004). Foxa2 regulates alveolarization and goblet cell hyperplasia. Development 131: 953-964.

WARD, H. and NICHOLAS, T. (1984). Alveolar type I and type II cells. Australian and New Zealand J. Med. 14: 731-734.

WARNER, B.B., STUART, L.A., PAPES, R.A. and WISPÉ, J.R. (1998). Functional and pathological effects of prolonged hyperoxia in neonatal mice. Am. J. Physiol.-Lung Cell. Molec. Physiol. 275: L110-L117.

YU, A.S., CHENG, M.H., ANGELOW, S., GUNZEL, D., KANZAWA, S.A., SCHNEEBERGER, E.E., FROMM, M. and COALSON, R.D. (2009). Molecular basis for cation selectivity in claudin-2-based paracellular pores: identification of an electrostatic interaction site. J Gen Physiol 133: 111-127. 


\section{Further Related Reading, published previously in the Int. J. Dev. Biol.}

Signaling pathways during maintenance and definitive endoderm differentiation of embryonic stem cells Lina Sui, Luc Bouwens and Josué K. Mfopou

Int. J. Dev. Biol. (2013) 57: 1-12

http://dx.doi.org/10.1387/ijdb.120115ls

Patterning parameters associated with the branching of the ureteric bud regulated by epithelial-mesenchymal interactions Yanfeng Lin, Shaobing Zhang, Juha Tuukkanen, Hellevi Peltoketo, Taina Pihlajaniemi and Seppo Vainio

Int. J. Dev. Biol. (2003) 47: 3-13

http://dx.doi.org/10.1387/ijdb.12653247

Abrogation of tumor necrosis factor-alpha converting enzyme inhibits embryonic lung morphogenesis in culture

$\mathrm{J}$ Zhao, H Chen, Y L Wang and D Warburton

Int. J. Dev. Biol. (2001) 45: 623-631

http://dx.doi.org/10.1387/ijdb.11460998

$\mathrm{N}$-cadherin protein distribution in normal embryos and in embryos carrying mutations in the homeobox gene Hoxa-4

A I Packer, V A Elwell, J D Parnass, K A Knudsen and D J Wolgemuth

Int. J. Dev. Biol. (1997) 41: 459-468

http://dx.doi.org/10.1387/ijdb.9240562

TTF-1/NKX2.1 up-regulates the in vivo transcription of nestin

Roberta Pelizzoli, Carlo Tacchetti, Paola Luzzi, Antonella Strangio, Grazia Bellese, Emanuela Zappia and Stefania Guazzi*

Int. J. Dev. Biol. (2008) 52: 55 - 62

doi: 10.1387/ijdb.062196rp

5 yr ISI Impact Factor $(2013)=2.879$
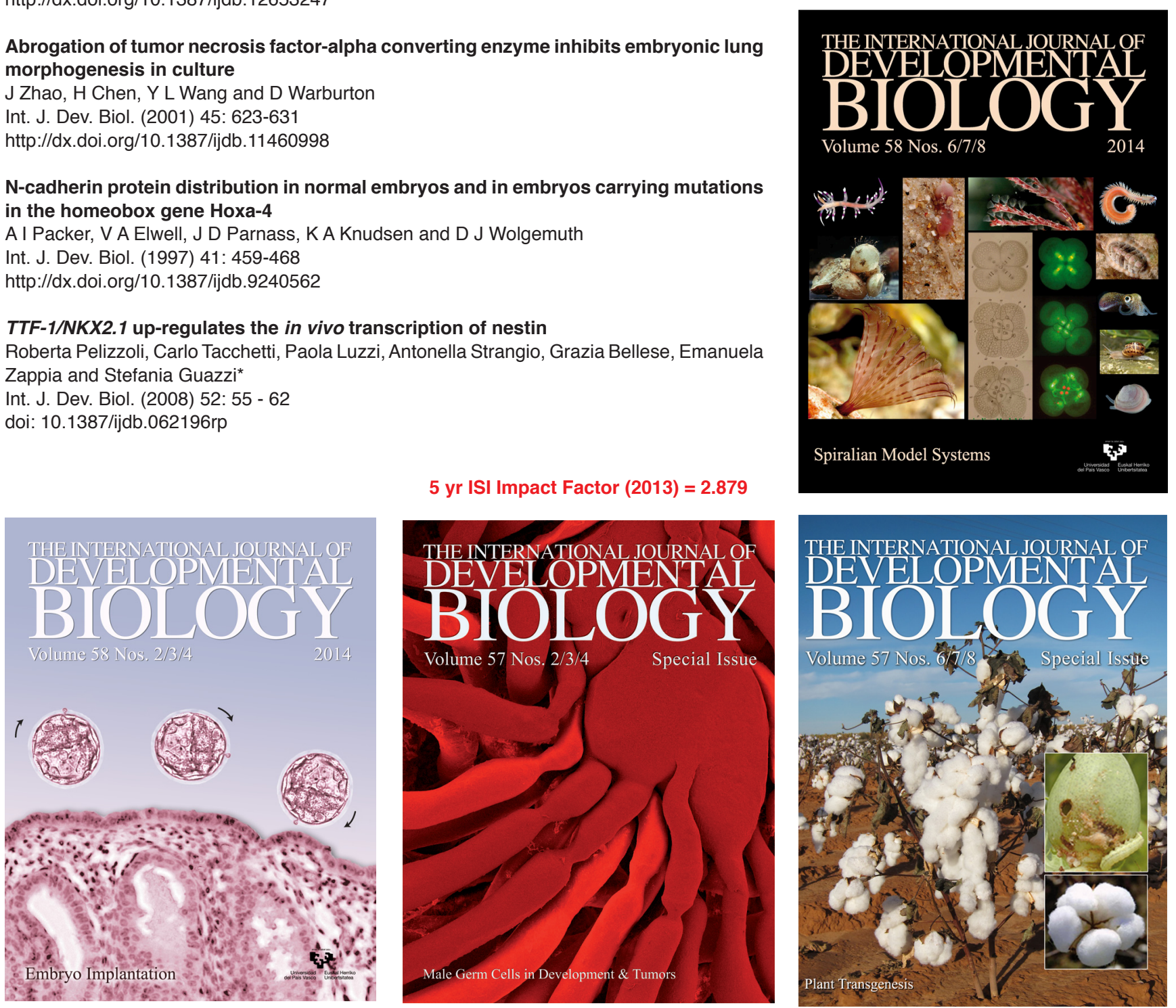